\title{
HPV16 E7 nucleotide variants found in cancer-free subjects af- fect E7 protein expression and transformation
}

\author{
Hong Lou1, Joseph F. Boland1, Robert Burk2, Meredith Yeager1, Nicolas Wentzensen3, Mark Schiffman3, Lisa \\ Mirabello3, Michael Dean4\# \\ 1 Cancer Genetics Research Laboratory, Division of Cancer Epidemiology and Genetics, Frederick National \\ Laboratory for Cancer Research, Rockville, MD USA \\ 2 Albert Einstein College of Medicine, Bronx, NY, USA \\ 3 Laboratory of Cancer Genetics, Division of Cancer Epidemiology and Genetics, National Cancer Institute, \\ Rockville, MD USA; \\ 4 Laboratory of Translational Genomics, Division of Cancer Epidemiology and Genetics, National Cancer \\ Institute, Rockville, MD USA \\ * Correspondence: Michael Dean, Ph.D., 9615 Medical Center Drive, Rm 3130, Laboratory of Translational \\ Genomics, National Cancer Institute, Rockville, MD 20850 USA. Tel: 240-760-6484, FAX: 301-402-3134 \\ (email: deanm@mail.nih.gov)
}

\begin{abstract}
The human papillomavirus (HPV) type 16 E7 oncogene is critical to carcinogenesis and highly conserved. Previous studies identified a preponderance of non-synonymous E7 variants amongst HPV16-positive cancer-free controls compared to those with cervical cancer. To investigate the function of E7 variants, we constructed full-length HPV16 E7 genes and tested variants at positions H9R, D21N, N29S, E33K, T56I, D62N, S63F, S63P, T64M, E80K, D81N, P92L, and P92S (found only in controls); D14E, N29H (CIN2), and P6L, H51N, R77S (CIN3). We determined the steadystate level of cytoplasmic and nuclear HPV16 E7 protein. All variants from the controls showed a reduced level of steady-state E7 protein, with 7/13 variants having deficient protein levels. In contrast, $2 / 3$ variants from the CIN3 precancer group had near-normal E7 levels. We assayed the activity of representative variants in stably transfected NIH3T3 cells. The H9R, E33K, P92L, and P92S variants found in control subjects had lower transforming activity than D14E and N29H variants (CIN2); and the R77S (CIN3) had activity only slightly reduced from wildtype E7. In addition, R77S and WT E7 caused increased migration of NIH3T3 cells in a wound-healing assay as compared with H9R, E33K, P92L, and P92S (controls) and D14E (CIN2). These data provide evidence that the E7 variants found in HPV16-positive cancer-free women are partially defective for transformation and cell migration further demonstrating the importance of fully active E7 in clinical cancer development
\end{abstract}

Keywords: HPV16, E7 variants, cervical cancer, transformation, wound healing, western blotting

\section{Introduction}

Cervical cancer is the second most common cancer among women worldwide and remains a clinical problem despite early detection and therapy [1]. The HPV16 E7 oncoprotein is essential for the normal viral life cycle and throughout the carcinogenic process from benign precursor lesions to invasive carcinoma [2]. HPV-induced carcinogenesis results from the deregulated expression of the HPV E6 and E7 oncoproteins and E6 and E7 function to establish and maintain a cellular environment that supports viral genome replication [3] . HPV does not encode enzymes for DNA replication; thus, viral genome synthesis uses cellular DNA replication enzymes. Aberrant cell proliferation is a crucial feature of cervical cancer. Cell cycle control is often disturbed; indeed, the p53 and pRB (retinoblastoma protein) tumor suppressor pathways are rendered dysfunctional in most cervical tumors $[4,5]$.

HPV16 E7 is a multifunctional protein that interacts with multiple cellular factors [6-10]. One of the main activities of E7 is to induce terminally differentiated cells to re- 
enter the cell cycle through disruption of the pRB/E2F system. Thus, E7 binds to and targets $\mathrm{pRB}$, which has an essential role in proliferation control by regulating the E2F transcription factor family affecting cell cycle progression, nucleotide synthesis, DNA replication, and apoptosis [11, 12].

HPV16 E7 is only 98-amino acids, yet it has a high number of binding targets [13]. The E7 protein consists of a C-terminal zinc finger-like domain (residues 38-98), a conserved region 3 (CR3) [14], and N-terminus (residues 1-37), CR1 (residues 1-15) and CR2 (residues 16-37) [14] domains. The CR1 and CR2 domains contain the binding motif for pRB linked by a proline-rich hinge region [15]. CR3 has a defined three-dimensional structure comprised of a unique $\beta 1 \beta 2 \alpha 1 \beta 3 \alpha 2$ fold that is not present in any other known zinc-binding protein $[16,17]$. HPV16 E7 also disrupts complexes between pRB and E2F or other related proteins in mouse cells and has been shown to transform established mouse cell lines $[18,19]$.

In a study of 5,570 HPV16 infected cervical precancer/cancer cases and controls (women with a benign HPV16 infection, defined as $\leq$ cervical intraepithelial neoplasia [CIN] grade 1 [CIN1], or an infection that was subsequently cleared), we observed significantly more E7 amino-acid changing variants in controls compared to cases [20]. The substitutions were in different domains of the E7 protein, and we hypothesized the variants suppress carcinogenesis. To test the ability of these variants to affect E7 function, we expressed the variants in multiple in vitro systems to determine steady-state protein expression, transformation, and migration ability.

\section{Materials and Methods}

Plasmid construction and antiHPV16 E7 antibodies. A synthetic full-length HPV16 A1 sequence was used as a template for preparing an HPV16 E7 construct with the primer set: RecNehI_HPV16-A1E7 Forward RecBamHI_HPV16-A1E7 Reverse (Table S1). The HPV16 E7 PCR product was cloned into the NheI and BamHI sites of the pcDNA3.1(+) IRES GFP vector (Addgene, Watertown, MA) using the CloneEZ® PCR Cloning Kit according to manufacturer's instructions (GenScript, Piscataway, NJ). E7 protein expression was confirmed by western blotting, with three positive control vectors from Addgene, CMV16 E7 (WT), CMV16 E7 C91S (MT1), CMV16 E7 del DLYC (MT2) in 293T (Table S2). In addition, we tested three commercial antibodies to detect HPV16 E7 expression 48h after transfection (Table S2). We found the antiHPV16 E7 (716-325) antibody (Santa Cruz, Dallas, TX) is more sensitive and specific for proteins extracted from the transfected 293T and cervical cancer cell lines, CaSki (HPV16), SiHa (HPV16), and HeLa (HPV18).

Mutant construction. The pcDNA3.1(+) IRES GFP-HPV16 E7 WT was used as a template to generate variant constructs using the CloneEZ® PCR Cloning (GenScript) and sitedirected mutagenesis methods (Agilent, Santa Clara, CA) according to the manufacturer's instructions $[22,23]$. The oligonucleotides used to generate the mutations are listed in Table S1. All resulting constructs were sequenced. The empty vector and wild-type E7 constructs were used as negative and positive controls.

Cell culture and transfection. The cancer cell lines CaSki (RRID: CVCL_1100), SiHa (RRID: CVCL_0032), HeLa (RRID: CVCL_0030) and HEK293T (RRID: CVCL_0063), as well as mouse fibroblast NIH-3T3 (RRID: CVCL_0594) cells, were obtained from ATCC and cultured according to the provider's instructions and were regularly tested for mycoplasma infection. CaSKi, SiHa, and NIH-3T3 cells have been validated by Identifiler (ThermoFisher, Waltham, MA) typing in the last three years. To determine HPV16 E7 protein expression in vitro, HeLa and 293T cells were seeded in 6 well plates at 2.5x10 cells per well and transfected after $48 \mathrm{~h}$ with 2 - $4 \mu \mathrm{g}$ of plasmid DNA using HilyMax 
(Dojindo, Kumamoto, Japan) transfection reagents according to the manufacturer's instructions [21][22]. At 48h after transfection, cells were harvested, and nuclear and cytoplasmic fractions separated using the CelLyticTM NuCLEAR Extraction Kit (Sigma-Aldrich, St. Louis, MO). Protein concentrations were determined with the Bio-Rad protein assay using the standard curve method (Bio-Rad Laboratories, Hercules, CA) [21][22].

Western blotting. Nuclear and cytoplasmic lysates were resolved on 10\% NuPAGE Novex Bis-Tris polyacrylamide gels (Invitrogen, Waltham, MA), transferred to a PVDF membrane, blocked at room temperature for two hours in 5\% milk in Tris-buffered saline with $0.1 \%$ Tween 20 (TBST), and then blotted. Western blot analysis was performed with the following antibodies: HPV16 E7 (716-325) mouse monoclonal antibody at 1:100 (Santa Cruz); and purified mouse anti-human retinoblastoma (protein Clone, G3-245) $(\mathrm{pRB})$ at 1:250 (BD Biosciences, Franklin Lakes, NJ). In addition, we selected rabbit monoclonal antibody at 1:1000 of TBP (D5G7Y) and beta-Tubulin (9F3) (Cell Signaling, Danvers, MA) as loading controls for nuclear and cytoplasmic proteins for WB, respectively.

Determination of morphological transformation. NIH3T3 cells stably transfected with the empty vector, wild type E7, and the indicated E7 variants were plated in $75 \mathrm{ml}$ flasks at an equal cell density in regular DMEM medium with G418 500 $\mathrm{\mu g} / \mathrm{ml}$. At $60 \%$ confluence, cell morphology was examined and imaged under a microscope with 10x and 20x magnification (Zeiss Axio, Carl Zeiss, Germany). NIH3T3 cells were used in an HPV16 transformation assay by examining anchorage-independent growth in soft agar. NIH3T3 cells were transfected with pcDNA3.1(+) IRES GFP-HPV16 E7 vectors as described above. At $48 \mathrm{~h}$ after transfection, cells were selected in a medium containing G418. After three weeks of selection, the stably transfected cells were seeded into soft agar in 96-well plates.

Anchorage-independent cell growth. We used the Cytoselect 96-well Cell Transformation Assay (Cell Biolabs, San Diego, CA) to assess colony formation according to the manufacturer's instructions. We counted live cells (variability $>95 \%$ ) using the Countess automated cell counter (Invitrogen, Waltham, MA), and each cell line was counted twice. The cells were seeded in soft agar at a density of $1 \times 10^{4}$ cells per well, and duplicate plates were tested using Quantitation of Anchorage-Independent Growth and colony formation. The colony formation was quantified by solubilizing the soft agar, lysing the cells, and incubating cell lysates with the CyQUANT GR Dye (Cell Biolabs) with the GLOMAX Multi Detection System (Promega, Madison, WI). In addition, the colonies were imaged under a microscope (Zeiss Axio, Carl Zeiss, Germany).

Wound healing assay. The migration of NIH3T3 stably transfected cells was monitored by the Lionheart FX Automated Live Cell Imager (BioTek, Winooski, VT), and cellular analysis of the kinetic images was performed using Gen5 software. Cells were grown in T75 flasks until they reached a confluency of $60-70 \%$. The cells were detached from the flask using phenol red-free TrypLE Express Trypsin (Gibco/Thermo Fisher). The method for cell counting was the same as described above, and cells were seeded in 24-well plates with 1.0x10 live cells per well. Cell monolayers were scratched using an Autoscratch machine (BioTek). The plate was placed in the Lionheart FX Automated Live Cell Imager in $5 \% \mathrm{CO}_{2}$ at $37^{\circ} \mathrm{C}$. The wound area was imaged every 2 hours over a period of 20 hours using a $4 x$ objective and phase contrast, and bright field illumination. The number of migrating cells into the wound area was counted using percent confluency within the wound area (www.biotek.com).

Statistical analysis. Mann-Whitney-U tests and unpaired two-tailed t-tests were performed using GraphPad Prism version 8 for Windows 10; P-values of $<0.05$ were regarded to be significant. 


\section{Results}

\subsection{HPV16 E7 mutagenesis and protein expression}

To test E7 protein expression and function of 18 specific E7 nucleotide variants, we generated expression constructs in the full-length HPV16 E7 open reading frame (ORF, 98 AAs) in the pcDNA3.1(+) IRES GFP vector. We constructed nine variants using the ClonEZ PCR cloning method, nine by site-directed mutagenesis, and all confirmed by DNA sequencing. Figure 1 shows the distribution of variants in this study and the E7 protein functional domains affected. Eleven variants were in CR3, and three and four variants in CR1 and CR2, respectively. A summary of all of the tested E7 mutants and their cancer association and protein expression is shown in Table $\mathbf{1 .}$

Figure 1

\section{HPV16 E7 variants in this study}

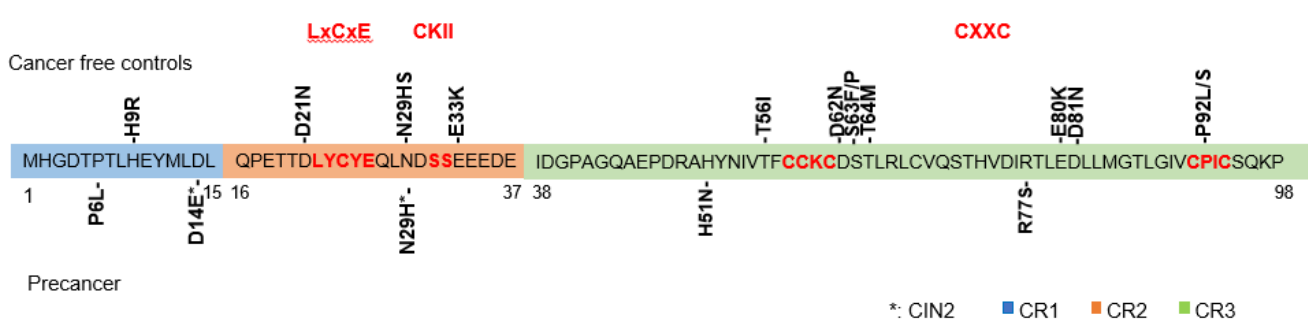

Figure 1. HPV16 E7 variants in this study. The single amino acid (AA) substitution mutants are shown in relation to functional domains. Variants from controls only are shown above the diagram and variants from pre-cancer below. The numbers 1 to number 98 indicate the amino acid position of the E7 protein. The LxCxE motif (AA 22-26); CKII motif (AA 31-32); and CXXC motifs (AA 58-61 and AA 91-94) are shown in red. The E7 functional domains include three conserved regions, CR1, CR2 and CR3. *: indicates cervical intraepithelial neoplasia 2 (CIN2).

Table 1. HPV16 E7 variants tested and associated nuclear protein levels of E7 and pRB

\begin{tabular}{|c|c|c|c|c|c|c|c|}
\hline Variants & $\begin{array}{c}\text { Origi } \\
\text { nal codon }\end{array}$ & Mutant codon & $\begin{array}{c}\text { Nucleotide } \\
\text { change }\end{array}$ & $\begin{array}{c}\text { Control } \\
\text { (no.) }\end{array}$ & $\begin{array}{c}\text { Precancer } \\
\text { (no.) }\end{array}$ & $E 7^{*}$ & $\mathrm{pRB}$ \\
\hline Wild type & & & & & & 1 & 1 \\
\hline H9R & CAT & CGT & $A>G$ & 1 & 0 & 0.8 & 0.9 \\
\hline D14E & GAT & GAA & $\mathrm{T}>\mathrm{A}$ & 0 & 1 CIN2 & 0.11 & 1.4 \\
\hline $\mathrm{D} 21 \mathrm{~N}$ & GAT & AAT & $G>A$ & 1 & 0 & 0.92 & 0.93 \\
\hline $\mathrm{N} 29 \mathrm{H}$ & AAT & CAT & $A>C$ & 0 & 1 CIN2 & 0.3 & 1.5 \\
\hline N29S & AAT & AGT & $A>G$ & 2 & 0 & 0.018 & 1.4 \\
\hline E33K & GAG & AAG & $G>A$ & 3 & 0 & 0.2 & 0.86 \\
\hline T56I & $A C C$ & ATC & $\mathrm{C}>\mathrm{T}$ & 1 & 0 & 0.55 & 1.4 \\
\hline D62N & GAC & AAC & $G>A$ & 1 & 0 & 0.11 & 1.2 \\
\hline
\end{tabular}




\begin{tabular}{|c|c|c|c|c|c|c|c|}
\hline S63F & TCT & TTT & $C>T$ & 1 & 0 & 0.1 & 1.0 \\
\hline S63P & TCT & CCT & $\mathrm{T}>\mathrm{C}$ & 1 & 0 & 0.3 & 1.2 \\
\hline T64M & ACG & ATG & $\mathrm{C}>\mathrm{T}$ & 1 & 0 & 0.11 & 1.3 \\
\hline E80K & GAA & AAA & $G>A$ & 1 & 0 & 0.26 & 1.0 \\
\hline D81N & GAC & AAC & $G>A$ & 1 & 0 & 0.42 & 0.95 \\
\hline P92L & $\mathrm{CCC}$ & СTC & $C>T$ & 1 & 0 & 0.1 & 1.2 \\
\hline P92S & $\operatorname{ccc}$ & TCC & $C>T$ & 2 & 0 & 0.68 & 1.2 \\
\hline P6L & CCT & CTT & $C>T$ & 1 & $1 \mathrm{CIN} 3$ & 1.1 & 1.5 \\
\hline $\mathrm{H} 51 \mathrm{~N}$ & CAT & AAT & $C>A$ & 6 & 3 CIN3/AIS & 0.46 & 1.1 \\
\hline R77S & CGT & AGT & $\mathrm{C}>\mathrm{A}$ & 1 & 1 AIS & 0.99 & 1.2 \\
\hline
\end{tabular}

To determine if the variants affect the level of steady-state E7 protein, we transfected them into HeLa, and 293T cells and nuclear and cytoplasmic proteins were detected by western blotting (WB). These cell lines were chosen because HeLa cells are derived from cervical cancer and have an intact pRB tumor suppressor pathway[23-26], and 293T is an HPV-negative cell line derived from a human fetus and has high transfectability.

The anti-HPV16 E7 (716-325) antibody was tested for sensitivity and specificity with $293 \mathrm{~T}$ transfected with three positive control vectors and in cervical cancer cell lines containing HPV16 (CaSki and SiHa) or HPV18 (HeLa) (Figure S1 and Table S2). The positive controls and our WT E7 vector displayed a correctly sized E7 protein (Figure S1A). Similarly, the HPV16 E7 antibody detected the endogenous HPV16 E7 protein in the cervical cancer cell lines CaSki (600 copies of HPV16) and SiHa (1-2 copies of HPV16), but not the HPV18 E7 protein in HeLa cells (Figure S1B). Therefore, the antiHPV16 E7 (716-325) antibody is sensitive and specific for HPV16 E7 protein, consistent with published data $[27,28]$.

\subsection{Reduced Protein expression levels of HPV16 E7 variants}

The HPV16 E7 protein has been previously localized to the cytoplasm and nucleus and observed as a dimer in the nucleus[29-33]. To determine the location of our HPV16 E7 WT and mutant proteins, we separated cytoplasmic and nuclear fractions and observed the E7 protein in both fractions in HeLa and 293T cells (Figure 2). For the 13 E7 variants previously identified in controls, seven (54\%), N29S, E33K, D62N, S63F, S63P, T64M, and P92L, showed deficient levels of steady-state E7 protein in the nucleus as compared with E7 WT protein, and the four remaining (36\%), T56I, E80K, D81N, and P92S, had moderately reduced levels (Figure 2A; Figure S2A). In 293T cells, 9 out of 10 (90\%) of the variants tested in E7 identified in controls also showed decreased E7 protein levels as compared to WT in both nuclear and cytoplasmic fractions (Figure 2B; Figure S2B).

Similarly, we found a severe to moderate reduction of E7 protein levels for the D14E, $\mathrm{N} 29 \mathrm{H}$ variants identified in CIN2 and H9R and E33K in controls, in either HeLa or 293T cells (Figure 2A, B; Figure S2A, B). Two of the three precancer variants tested, P6L and R77S, showed E7 levels similar to WT E7, and H51N showed moderately reduced levels (Figure 2A, Table 1). P6L is adjacent to another mutation (T5K) with transforming potential like E7 WT [34]. 
Figure 2A.

HPV16 E7 protein levels in transfected HeLa cells

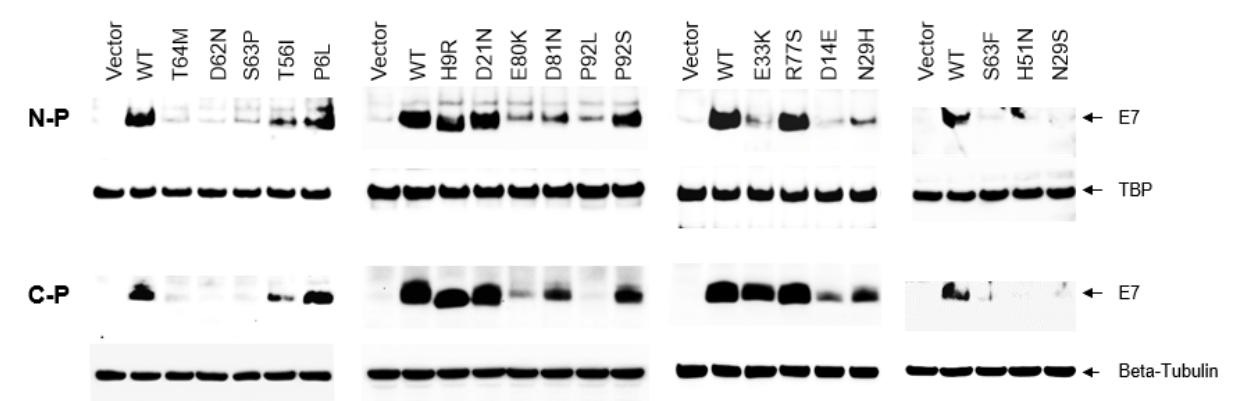

Figure 2B. HPV16 E7 protein levels in 293T cells
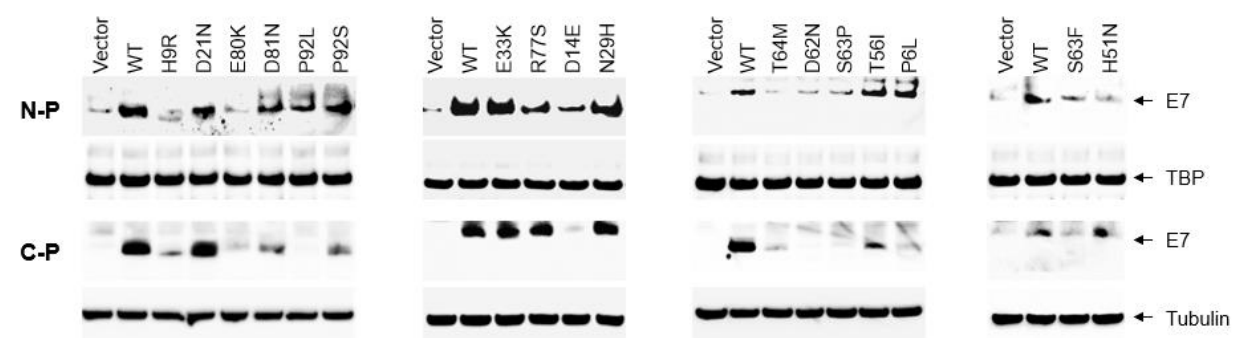

Figure 2. Western blot analyses of HPV16 E7 from HeLa (A) and 293T (B) cells transfected with empty vector, wild-type and indicated mutant E7 constructs. The position of the E7 and tubulin-binding protein (TBP) are indicated. N-P and C-P represent the nuclear and cytoplasm proteins, respectively. Statistical analysis of three independent experiments is shown in Figure S2.

3.3. HPV16 E7 variants not associated with cancer have a more normal cell morphology and reduced anchorage-independent growth in NIH3T3 cells

To test the ability of HPV16 E7 transfected cells to transform cells, we chose a representative subset of eight of the mutant-expressing cells (6 from the control group [H9R, D14E, D21N, E33K, P92L, P92S] and two from the CIN2 or CIN3 precancer groups [N29H, R77S]), and assessed cell morphology and growth in NIH3T3 cells. For these assays, we created stably transfected NIH3T3 cell lines of each of the eight mutants. As shown in Figure 3A, E7 WT cells displayed a spindle-shaped morphology and more rounded, mitotic cells. However, the control variant (H9R, P92S) cells and empty vectortransfected cells had a near-normal morphology, and E33K cells displayed an intermediate phenotype.

To determine the effects of HPV16 E7 variants on cellular transformation, we assessed growth and colony size in soft agar. Wild-type NIH3T3 cells or empty vectortransfected cells display few colonies of small size, whereas WT E7-transfected cells show more colonies and of a larger size (Figure 3B, Figure S3). We used a soft agar growth assay, quantitated with a fluorescent dye, to assess abnormal cell growth. Figure $3 \mathrm{C}$ shows the quantification of the colony size results, including a WT E7 control and a mutation in the CxxC domain known to be non-functional [35]. All four variants with significantly reduced (50-60\%) transforming activity (H9R, E33K, P92L, and P92S) come from control subjects. Two of the three variants with $70-80 \%$ transforming activity are from CIN2 subjects and the R77S variant, from a CIN3 precancer subject, has similar 
activity as the WT (Figure 3C). Therefore, 80\% of the control mutants tested caused significantly reduced anchorage-independent growth activity.

Figure 3A.

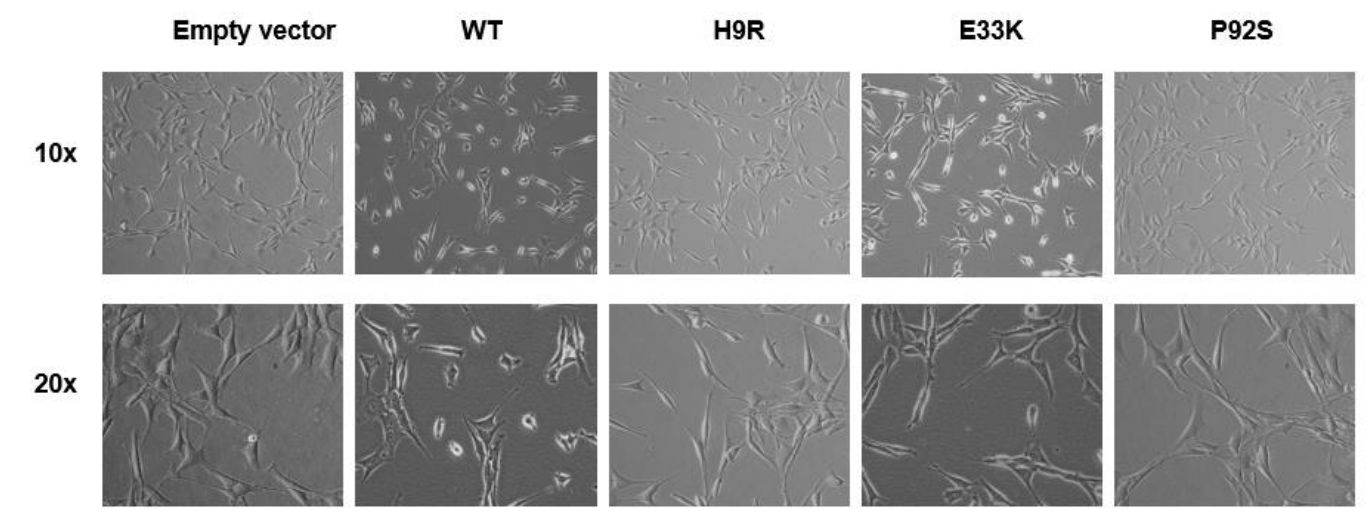

Figure 3B.

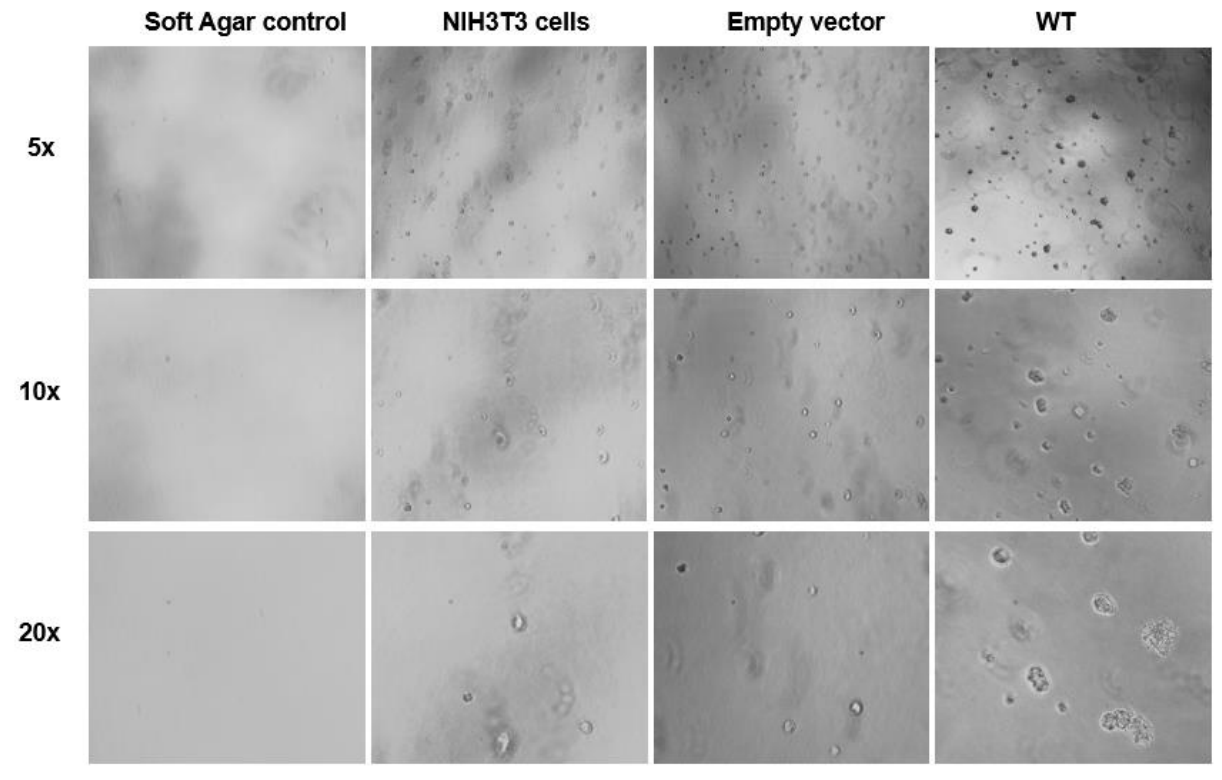

Figure 3C.

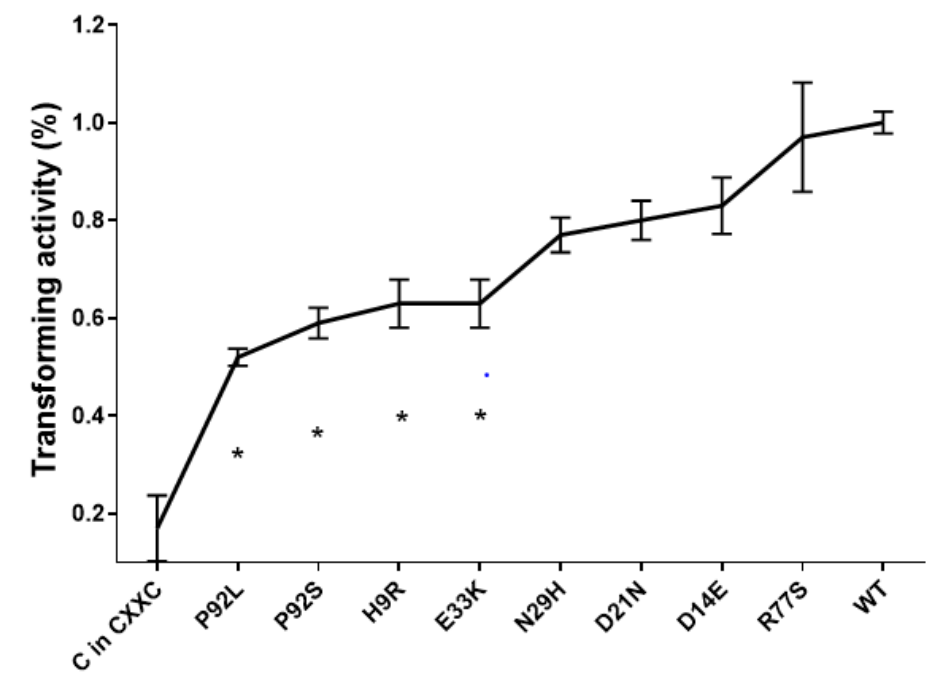


Figure 3. Morphology and anchorage independent growth of NIH3T3 transfected with HPV16 E7 WT and variants types. (A). Morphology of NIH3T3 cells stably expressing empty vector, WT and the indicated variant types. Morphology of stable NIH3T3 cells (10x, 20x). (B). Anchorage independent cell growth of HPV16 E7 controls on soft agar. NIH3T3 cells stably transfected with the empty vector, WT E7 are shown. Colonies formed 20 days and were imaged with 5x, 10x, 20x magnification. See Figure S3 for images of variants. (C). Quantitation of anchorage independent cell growth of HPV16 E7 variants on soft agar. HPV16 E7 WT cell growth was used as the $100 \%$ value. The colony formation of stable NIH3T3 cells was quantified using the fluorescent CyQUANT GR Dye. $C$ in CXXC indicate Cysteine (C) changed to glycine in the CXXC motif at positions 58 and 91 [35]. ${ }^{*}=\mathrm{P}<0.05$ for comparison with WT cells.

\subsection{HPV16 E7 variants display decreased NIH3T3 cell migration}

To determine the effect of a representative subset of seven variants (those noted above, excluding $\mathrm{N} 29 \mathrm{H}$ ) on cell migration in a wound-healing assay, we performed automated scratches of a cell monolayer, monitoring cell migration every two hours, over 20 hours. HPV16 E7 WT and R77S (from CIN3 precancer) NIH3T3 cells showed increased migration into the wound area as compared with H9R (CIN2), E33K (CIN2), P92L (Control), and P92S (Control) and moderately increased compared with D21N (Control) and D14E (CIN2; Figure 4). The percentage of cell confluency in the wound area was calculated from images taken every 2 hours in a LionHeart FX Automated Live Cell Imager in triplicate. Analysis of the time course of wound closure showed that WT and R77S (CIN3) cells displayed significantly increased migration compared to H9R, E33K, P92L, and P92S, from controls. In addition, H9R, P92L, and P92S showed somewhat faster wound closure than empty vector-transfected cells, and in this assay, the E33K variant was indistinguishable from the empty vector. These results confirm that four of the six HPV16 E7 control or CIN2 group mutants have decreased activity of the E7 protein, as measured by NIH3T3 cell migration (Figure 4B). The data from all the tested E7 mutants, associated phenotypes, and assay results are summarized in Table 2. Using extended follow-up data from these individuals (see [20]), we found that 5/13 $(38 \%)$ of the subjects from the control group were HPV negative on a subsequent visit and therefore cleared their HPV16 infection (Table 2).
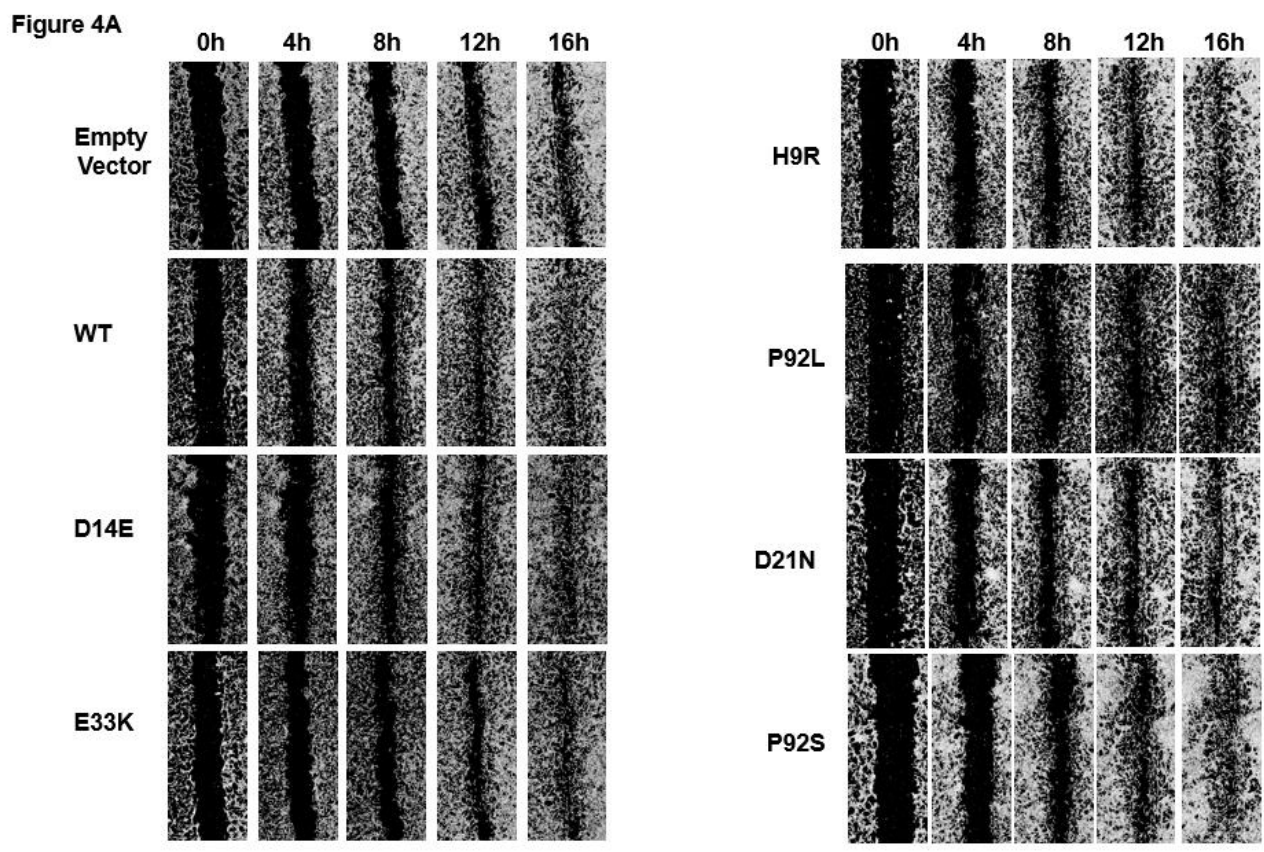


\section{Figure 4B}

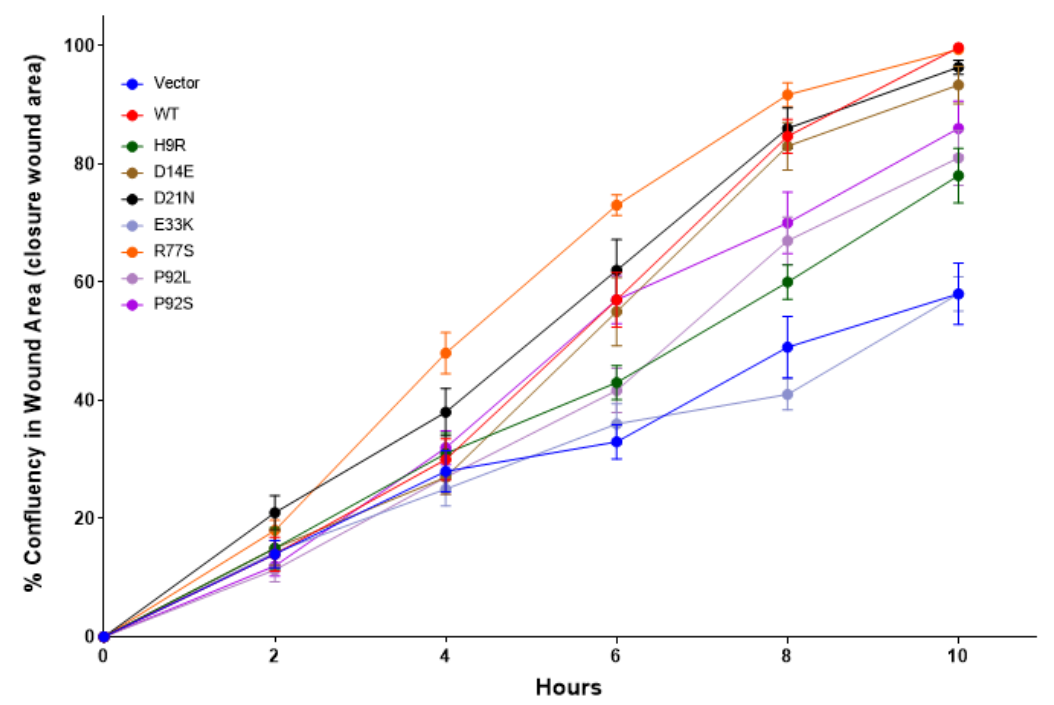

Figure 4. Migration of stably transfected NIH3T3 cell lines in a wound healing (scratch) assay. Stable NIH3T3 cell clones were plated onto 24-well plates. Wounds in the monolayer were created with an automated tool, plates were washed to remove detached cells and closure of the wound followed to $24 \mathrm{hr}$ with hourly imaging in a BioTek Lionheart FX Automated Live Cell Imager. Images are shown in Panel A, and quantitation over time in Panel B. At 10h: WT vs Vector $(\mathrm{p}=0.0079)$; WT-H9R $(\mathrm{p}=0.029)$; WT-E33K $(\mathrm{p}=0.0079) ;$ WT-D14E $(\mathrm{p}>0.05) ;$ WT-P92L $(\mathrm{p}=0.029)$ WT-P92S $(\mathrm{p}=0.033)$.

Table 2. Summary of the HPV16 E7 variants, protein expression and functional assays

\begin{tabular}{ccccccc}
\hline Construct & Cervical disease & $\begin{array}{c}\text { HPV16 clear- } \\
\text { ance }\end{array}$ & $\begin{array}{c}\text { Function Do- } \\
\text { main }\end{array}$ & $\begin{array}{c}\text { Nuclear } \\
\text { Protein }\end{array}$ & $\begin{array}{c}\text { Transforming activity (\% } \\
\text { growth in soft agar) }\end{array}$ & Wound Healing \\
\hline Vector & & & & & & + \\
\hline D81N & Control & & CR3 & ++ & NA & NA \\
E80K & Control & & CR3 & ++ & NA & NA \\
P92S & Control & & CR3 & ++ & ++ & ++ \\
S63F & Control & & CR3 & + & NA & NA \\
T56I & Control & & CR3 & ++ & NA & NA \\
T64M & Control & & CR3 & + & NA & NA \\
D21N & Control & yes & CR2 & +++ & +++ & ++ \\
D62N & Control & & CR3 & + & NA & NA \\
P92L & Control & yes & CR3 & + & ++ & ++ \\
S63P & Control & yes & CR3 & + & NA & NA \\
E33K & Control & yes & CR2 & + & ++ & + \\
H9R & Control & & CR1 & +++ & ++ & ++ \\
N29S & Control, CIN2 & yes (both) & CR2 & + & NA & NA \\
\hline N29H & CIN2 & & CR2 & ++ & +++ & NA \\
D14E & CIN2 & & CR1 & ++ & +++ & +++ \\
\hline H51N & CIN3/AIS & & CR3 & ++ & NA & NA \\
P6L & CIN3 & & CR1 & ++++ & +++ & NA \\
R77S & AlS & & CR3 & ++++ & +++ \\
\hline
\end{tabular}




\section{Wild type}

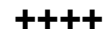

$++++$

CIN2, cervical intraepithelial neoplasia grade 2; CIN3, cervical intraepithelial neoplasia grade 3; AIS, adenocarcinoma in situ. *, also in HNSCC [36]. Green highlighting indicates a significant decrease in activity.

\section{Discussion}

The E7 oncogene is one of the two most important genes contributing to the cellular transformation and oncogenicity of high-risk HPV types. As HPV16 is the most carcinogenic HPV type, accounting for over half of all cervical cancer cases worldwide, it is critical to understand the activities of this protein. There are now multiple reports demonstrating a lack of genetic variation in the E7 protein in HPV16 isolates from cervical precancer and cancer, as well as head and neck cancer $[20,36]$. The most extensive study of 5,570 HPV16 genomes demonstrated a 6 to 7-fold increase in HPV16 E7 variants in isolates from women without cancer [20]. Our data presented here provides a partial mechanistic explanation for this observation.

The aim of this study was to assess the role of E7 amino acid variants previously identified [20] in controls and precancer cases to understand how these alterations may affect cervical carcinogenesis. The approaches used were to transfect HeLa, 293T, and NIH3T3 cells with expression vectors containing either WT or mutant forms of E7 to test whether these variants altered the steady-state levels of E7 protein in the cytoplasm and nucleus, as well as their efficiency to alter morphology, anchorage-independent growth, and migration in NIH3T3 cells. We found that most of the E7 variants identified in controls have lower steady-state protein levels, in both the cytoplasm and the nucleus, in a cervical cancer cell line (HeLa) and non-cervical cells (293T). We have not determined whether the lower protein levels are due to alterations in translation, stability, or protein degradation. Still, the reduced levels, especially in the nucleus, are consistent with a reduced transforming ability.

The level of E7 protein does not always reflect the transforming activity of the protein, and we, therefore, used multiple methods to assess E7 activity (data summarized in Table 2). Anchorage-independent growth is considered the most informative in vitro assay to detect transformation activity [37]. Several mutants found exclusively in controls (H9R, E33K, P92L, and P92S) show 30-50\% lower transforming activity, whereas the D14E and R77S mutants found in precancers have $80-100 \%$ activity in this assay. E33K is in the CR2 domain and is immediately downstream of the two known CKII phospho-acceptor sites at S31 and S32 [38]. Its reduced transforming activity could be due to an alteration in phosphorylation. However, we also found that the E33K mutant appears to be defective in nuclear localization in HeLa cells, and it's in a region known to be critical for E7 localization to the nucleus (aa 1-37) [39]. The P92L and P92S mutants affect a conserved proline inside the C-terminal CXXC motif and may cause subtle alterations in E7 structure or stability [40].

The D14E mutant, seen in a single CIN2 subject, has a low steady-state protein level but a modestly reduced activity $(\sim 80 \%)$ in the soft agar assay. Aspartic and glutamic acid are similarly charged amino acids, and this alteration may only slightly affect E7 activity. Likewise, the other alterations identified in CIN2 or CIN3 precancer (P6L, N29H, $\mathrm{H} 51 \mathrm{~N}$, and R77S) would appear to be residues that can tolerate change. Gillison et al. [36] identified an H51N containing HPV16 isolate in a head and neck tumor, consistent with this variant being compatible with cancer.

We also assessed the activity of stably transformed NIH3T3 cells to migrate in culture using the wound-healing (scratch) assay. Using an instrument that automatically generates uniform scratches and continuously monitors cell growth over 20 hours could 
show a dramatic difference between NIH3T3 cells containing empty vectors and those with the wild-type E7 construct. Scratch assays have been used in monitoring oncoprotein E6 and E7 function and demonstrate that HPV16 oncoproteins promote cell migration and invasion [40,41]. Still, to our knowledge, this is the first study to employ the assay to study E7 mutants in NIH3T3 cells. The wound-healing activity of the E7 mutants gave very similar results as the anchorage-independent growth assay, with R77S showing activity equal (or perhaps stronger) than wild type; D14E and N29S slightly impaired E33K with highly impaired cell migration.

Interestingly, the previous finding that an alteration of the highly conserved arginine at residue 77 to glutamic acid (R77E) [42] has a high steady-state protein level and promotes migration equal to or better than wild type. Furthermore, R77 is in an alphahelical domain and does not directly touch pRB or E2F1, and R77E increases E7 pRB binding capacity [16]. Therefore, the effect of the R77S variant in our study on pRB binding would be interesting to explore further.

It is noteworthy that no variants in functional or structural E7 motifs, including the conserved residues of the LxCXE and CXXC motifs, have been found in patients. Mutations in these residues, C24G, E26G for LxCxE, and C58G and C91G for CXXC, dramatically reduce or eliminate E7 transforming activity [35, 43, 44]. E7 is a zinc-binding protein, and the cysteine residues in the zinc-finger binding domain (C58, C59, C61, C91, C94), known to be essential for E7 function. Therefore, it is likely that the variants identified in actively infected, cancer-free patients, do not involve amino acid residues of E7 that are essential for function. However, many of the variants found in controls are in amino acids in CR3 (P92L/S) or near the CXXC motifs (T56I, D62N, S63P/F, T64M) (Figure 1). This result suggests that alterations of residues near the CXXC motifs alter the structure of E7 and partially reduce function. Other variants near the LxCxE and CKII motifs partially reduce cellular oncogenicity $[35,43,44]$. It is also possible that some E7 variants produced peptides that are recognized by $\mathrm{T}$ cells, leasing to viral clearance. The precise molecular mechanism of action of variants of E7 in cervical cancer development needs to be further explored [16, 40, 41].

The limitations of this study are that we did not determine the molecular basis for the reduced steady-state level of protein and performed functional analyses on a subset of the variants. In addition, our functional assays involve NIH3T3 mouse fibroblast cells, and it would be informative to repeat these results using viral constructs in primary cells or an infectious model of HPV $[35,43,44]$. However, the data are consistent with the paradigm that many amino acid-altering E7 variants in controls have attenuated migration and transforming ability.

In conclusion, cervical cancer development appears to require the continuous activity of a fully functional E7 protein. Our genetic epidemiologic data previously demonstrated that variation in E7 is poorly tolerated [21], and here our results suggest that even a 50\% inhibition of E7 activity would render HPV defective for oncogenesis. These alterations may generate an HPV16 protein similar to low-risk HPV E7 proteins that are less efficient in binding and degrading $p R B$ proteins $[45,46]$. Our results suggest that inhibitors of HPV16 E7 expression, stability, or activity could play a role in cervical cancer prevention or treatment.

Supplementary Materials: The following are available online at www.mdpi.com/xxx/s1, Figure S1: Detection of HPV16 E7 expression level in transfected 293T cells and cervical cancer cell lines using antiHPV16 E7 (716-325) antibody. Figure S2, Western blot analyses of HPV16 E7 from HeLa cells transfected with empty vector, wild-type and mutant E7 constructs. Table S1: Oligonucleotide primers, Table S2: HPV16 E7 pcDNA clones and antibodies tested. 


\begin{abstract}
Author Contributions “Conceptualization, M.D., H.L., L.M.; methodology, H.L.; investigation, H.L; resources, M.D.; data curation, L.M.; writing-original draft preparation, M.D., H.L.; writing-review and editing, M.D., H.L., J.F.B., R.B., M.Y., N.W., L.M.; supervision, M.D.; funding acquisition, M.D. All authors have read and agreed to the published version of the manuscript.

Funding: This research was funded by the Intramural Program of the National Institutes of Health, National Cancer Institute. The content of this publication does not necessarily reflect the views or policies of the Department of Health and Human Services, nor does mention of trade names, commercial products, or organizations imply endorsement by the U.S. government.
\end{abstract}

Acknowledgments: The authors would like to thank Reema Vazirani for assistance with the Gen5 software, and Dr. Seth Brodie for useful discussions.

Conflicts of Interest: The authors declare no conflict of interest.

\title{
References
}

1. Schiffman, M.; Castle, P. E., The promise of global cervical-cancer prevention. N Engl J Med 2005, 353, (20), 2101-4.

2. Bodily, J. M.; Mehta, K. P.; Cruz, L.; Meyers, C.; Laimins, L. A., The E7 open reading frame acts in cis and in trans to mediate differentiation-dependent activities in the human papillomavirus type 16 life cycle. J Virol 2011, 85, (17), 8852-62.

3. Stubenrauch, F.; Laimins, L. A., Human papillomavirus life cycle: active and latent phases. Semin Cancer Biol 1999, 9, (6), 379-86.

4. Massimi, P.; Banks, L., Repression of p53 transcriptional activity by the HPV E7 proteins. Virology 1997, 227, (1), 255-9.

5. Moody, C. A.; Laimins, L. A., Human papillomavirus oncoproteins: pathways to transformation. Nat Rev Cancer 2010, 10, (8), 550-60.

6. Brehm, A.; Nielsen, S. J.; Miska, E. A.; McCance, D. J.; Reid, J. L.; Bannister, A. J.; Kouzarides, T., The E7 oncoprotein associates with Mi2 and histone deacetylase activity to promote cell growth. EMBO J 1999, 18, (9), 2449-58.

7. Bodily, J. M.; Mehta, K. P.; Laimins, L. A., Human papillomavirus E7 enhances hypoxia-inducible factor 1mediated transcription by inhibiting binding of histone deacetylases. Cancer Res 2011, 71, (3), 1187-95.

8. Berezutskaya, E.; Bagchi, S., The human papillomavirus E7 oncoprotein functionally interacts with the S4 subunit of the 26 S proteasome. J Biol Chem 1997, 272, (48), 30135-40.

9. White, E. A.; Sowa, M. E.; Tan, M. J.; Jeudy, S.; Hayes, S. D.; Santha, S.; Munger, K.; Harper, J. W.; Howley, P. M., Systematic identification of interactions between host cell proteins and $E 7$ oncoproteins from diverse human papillomaviruses. Proc Natl Acad Sci U S A 2012, 109, (5), E260-7.

10. Avvakumov, N.; Torchia, J.; Mymryk, J. S., Interaction of the HPV E7 proteins with the pCAF acetyltransferase. Oncogene 2003, 22, (25), 3833-41.

11. Helin, K.; Lees, J. A.; Vidal, M.; Dyson, N.; Harlow, E.; Fattaey, A., A cDNA encoding a pRB-binding protein with properties of the transcription factor E2F. Cell 1992, 70, (2), 337-50.

12. Dimova, D. K.; Dyson, N. J., The E2F transcriptional network: old acquaintances with new faces. Oncogene 2005, 24, (17), 2810-26.

13. Pim, D.; Banks, L., Interaction of viral oncoproteins with cellular target molecules: infection with high-risk vs low-risk human papillomaviruses. APMIS 2010, 118, (6-7), 471-493.

14. Phelps, W. C.; Yee, C. L.; Munger, K.; Howley, P. M., The human papillomavirus type 16 E7 gene encodes transactivation and transformation functions similar to those of adenovirus E1A. Cell 1988, 53, (4), 539-47. 
15. Chemes, L. B.; Glavina, J.; Alonso, L. G.; Marino-Buslje, C.; de Prat-Gay, G.; Sanchez, I. E., Sequence evolution of the intrinsically disordered and globular domains of a model viral oncoprotein. PLoS One 2012, 7, (10), e47661.

16. Liu, X.; Clements, A.; Zhao, K.; Marmorstein, R., Structure of the human Papillomavirus E7 oncoprotein and its mechanism for inactivation of the retinoblastoma tumor suppressor. J Biol Chem 2006, 281, (1), 578-86.

17. Ohlenschlager, O.; Seiboth, T.; Zengerling, H.; Briese, L.; Marchanka, A.; Ramachandran, R.; Baum, M.; Korbas, M.; Meyer-Klaucke, W.; Durst, M.; Gorlach, M., Solution structure of the partially folded high-risk human papilloma virus 45 oncoprotein E7. Oncogene 2006, 25, (44), 5953-9.

18. Kanda, T.; Furuno, A.; Yoshiike, K., Human papillomavirus type 16 open reading frame E7 encodes a transforming gene for rat 3Y1 cells. J Virol 1988, 62, (2), 610-3.

19. Vousden, K. H.; Doniger, J.; DiPaolo, J. A.; Lowy, D. R., The E7 open reading frame of human papillomavirus type 16 encodes a transforming gene. Oncogene Res 1988, 3, (2), 167-75.

20. Mirabello, L.; Yeager, M.; Yu, K.; Clifford, G. M.; Xiao, Y.; Zhu, B.; Cullen, M.; Boland, J. F.; Wentzensen, N.; Nelson, C. W.; Raine-Bennett, T.; Chen, Z.; Bass, S.; Song, L.; Yang, Q.; Steinberg, M.; Burdett, L.; Dean, M.; Roberson, D.; Mitchell, J.; Lorey, T.; Franceschi, S.; Castle, P. E.; Walker, J.; Zuna, R.; Kreimer, A. R.; Beachler, D. C.; Hildesheim, A.; Gonzalez, P.; Porras, C.; Burk, R. D.; Schiffman, M., HPV16 E7 Genetic Conservation Is Critical to Carcinogenesis. Cell 2017, 170, (6), 1164-1174 e6.

21. Lou, H.; Villagran, G.; Boland, J. F.; Im, K. M.; Polo, S.; Zhou, W.; Odey, U.; Juarez-Torres, E.; MedinaMartinez, I.; Roman-Basaure, E.; Mitchell, J.; Roberson, D.; Sawitzke, J.; Garland, L.; Rodriguez-Herrera, M.; Wells, D.; Troyer, J.; Pinto, F. C.; Bass, S.; Zhang, X.; Castillo, M.; Gold, B.; Morales, H.; Yeager, M.; Berumen, J.; Alvirez, E.; Gharzouzi, E.; Dean, M., Genome Analysis of Latin American Cervical Cancer: Frequent Activation of the PIK3CA Pathway. Clin Cancer Res 2015, 21, (23), 5360-70.

22. Lou, H.; Li, H.; Yeager, M.; Im, K.; Gold, B.; Schneider, T. D.; Fraumeni, J. F., Jr.; Chanock, S. J.; Anderson, S. K.; Dean, M., Promoter variants in the MSMB gene associated with prostate cancer regulate MSMB/NCOA4 fusion transcripts. Hum Genet 2012, 131, (9), 1453-66.

23. Psyrri, A.; DeFilippis, R. A.; Edwards, A. P.; Yates, K. E.; Manuelidis, L.; DiMaio, D., Role of the retinoblastoma pathway in senescence triggered by repression of the human papillomavirus E7 protein in cervical carcinoma cells. Cancer Res 2004, 64, (9), 3079-86.

24. Baker, C. C.; Phelps, W. C.; Lindgren, V.; Braun, M. J.; Gonda, M. A.; Howley, P. M., Structural and transcriptional analysis of human papillomavirus type 16 sequences in cervical carcinoma cell lines. J Virol 1987, 61, (4), 962-71.

25. Wrede, D.; Tidy, J. A.; Crook, T.; Lane, D.; Vousden, K. H., Expression of RB and p53 proteins in HPV-positive and HPV-negative cervical carcinoma cell lines. Mol Carcinog 1991, 4, (3), 171-5.

26. Wells, S. I.; Francis, D. A.; Karpova, A. Y.; Dowhanick, J. J.; Benson, J. D.; Howley, P. M., Papillomavirus E2 induces senescence in HPV-positive cells via pRB- and p21(CIP)-dependent pathways. EMBO J 2000, 19, (21), 5762-71.

27. Jenison, S. A.; Yu, X. P.; Valentine, J. M.; Koutsky, L. A.; Christiansen, A. E.; Beckmann, A. M.; Galloway, D. A., Evidence of prevalent genital-type human papillomavirus infections in adults and children. J Infect Dis 1990, 162, (1), 60-9.

28. Tindle, R. W.; Smith, J. A.; Geysen, H. M.; Selvey, L. A.; Frazer, I. H., Identification of B epitopes in human papillomavirus type 16 E7 open reading frame protein. J Gen Virol 1990, 71 ( Pt 6), 1347-54.

29. Greenfield, I.; Nickerson, J.; Penman, S.; Stanley, M., Human papillomavirus 16 E7 protein is associated with the nuclear matrix. Proc Natl Acad Sci U S A 1991, 88, (24), 11217-21. 
30. Huh, K. W.; DeMasi, J.; Ogawa, H.; Nakatani, Y.; Howley, P. M.; Munger, K., Association of the human papillomavirus type 16 E7 oncoprotein with the 600-kDa retinoblastoma protein-associated factor, p600. Proc Natl Acad Sci U S A 2005, 102, (32), 11492-7.

31. Ressler, S.; Scheiden, R.; Dreier, K.; Laich, A.; Muller-Holzner, E.; Pircher, H.; Morandell, D.; Stein, I.; Viertler, H. P.; Santer, F. R.; Widschwendter, A.; Even, J.; Jansen-Durr, P.; Capesius, C.; Zwerschke, W., High-risk human papillomavirus E7 oncoprotein detection in cervical squamous cell carcinoma. Clin Cancer Res 2007, 13, (23), 7067-72.

32. Nguyen, C. L.; Munger, K., Human papillomavirus E7 protein deregulates mitosis via an association with nuclear mitotic apparatus protein 1. J Virol 2009, 83, (4), 1700-7.

33. Zatsepina, O.; Braspenning, J.; Robberson, D.; Hajibagheri, M. A.; Blight, K. J.; Ely, S.; Hibma, M.; Spitkovsky, D.; Trendelenburg, M.; Crawford, L.; Tommasino, M., The human papillomavirus type 16 E7 protein is associated with the nucleolus in mammalian and yeast cells. Oncogene 1997, 14, (10), 1137-45.

34. Fujinaga, Y.; Okazawa, K.; Nishikawa, A.; Yamakawa, Y.; Fukushima, M.; Kato, I.; Fujinaga, K., Sequence variation of human papillomavirus type $16 \mathrm{E} 7$ in preinvasive and invasive cervical neoplasias. Virus Genes 1994, 9, (1), 85-92.

35. Edmonds, C.; Vousden, K. H., A point mutational analysis of human papillomavirus type 16 E7 protein. $J$ Virol 1989, 63, (6), 2650-6.

36. Gillison, M. L.; Akagi, K.; Xiao, W.; Jiang, B.; Pickard, R. K. L.; Li, J.; Swanson, B. J.; Agrawal, A. D.; Zucker, M.; Stache-Crain, B.; Emde, A. K.; Geiger, H. M.; Robine, N.; Coombes, K. R.; Symer, D. E., Human papillomavirus and the landscape of secondary genetic alterations in oral cancers. Genome Res 2019, 29, (1), 117.

37. Androphy, E. J.; Schiller, J. T.; Lowy, D. R., Identification of the protein encoded by the E6 transforming gene of bovine papillomavirus. Science 1985, 230, (4724), 442-5.

38. Firzlaff, J. M.; Galloway, D. A.; Eisenman, R. N.; Luscher, B., The E7 protein of human papillomavirus type 16 is phosphorylated by casein kinase II. New Biol 1989, 1, (1), 44-53.

39. Knapp, A. A.; McManus, P. M.; Bockstall, K.; Moroianu, J., Identification of the nuclear localization and export signals of high risk HPV16 E7 oncoprotein. Virology 2009, 383, (1), 60-8.

40. Hu, D.; Zhou, J.; Wang, F.; Shi, H.; Li, Y.; Li, B., HPV-16 E6/E7 promotes cell migration and invasion in cervical cancer via regulating cadherin switch in vitro and in vivo. Arch Gynecol Obstet 2015, 292, (6), 1345-54.

41. Chuerduangphui, J.; Pientong, C.; Chaiyarit, P.; Patarapadungkit, N.; Chotiyano, A.; Kongyingyoes, B.; Promthet, S.; Swangphon, P.; Wongjampa, W.; Ekalaksananan, T., Effect of human papillomavirus 16 oncoproteins on oncostatin M upregulation in oral squamous cell carcinoma. Med Oncol 2016, 33, (8), 83.

42. Todorovic, B.; Massimi, P.; Hung, K.; Shaw, G. S.; Banks, L.; Mymryk, J. S., Systematic analysis of the amino acid residues of human papillomavirus type 16 E7 conserved region 3 involved in dimerization and transformation. J Virol 2011, 85, (19), 10048-57.

43. Caldeira, S.; de Villiers, E. M.; Tommasino, M., Human papillomavirus E7 proteins stimulate proliferation independently of their ability to associate with retinoblastoma protein. Oncogene 2000, 19, (6), 821-6.

44. Lam, E. W.; Morris, J. D.; Davies, R.; Crook, T.; Watson, R. J.; Vousden, K. H., HPV16 E7 oncoprotein deregulates B-myb expression: correlation with targeting of p107/E2F complexes. EMBO J 1994, 13, (4), 871-8.

45. Storey, A.; Pim, D.; Murray, A.; Osborn, K.; Banks, L.; Crawford, L., Comparison of the in vitro transforming activities of human papillomavirus types. EMBO J 1988, 7, (6), 1815-20.

46. Pim, D.; Banks, L., Interaction of viral oncoproteins with cellular target molecules: infection with high-risk vs low-risk human papillomaviruses. APMIS 2010, 118, (6-7), 471-93. 
